Kong. Res. J. 2(2) : 1-5, 2015

ISSN 2349-2694

Kongunadu Arts and Science College, Coimbatore.

\title{
BIOMIMETIC SYNTHESIS AND CHARACTERIZATION OF SILVER NANOPARTICLES (AGNPS) USING VINCA ROSEA AQUEOUS EXTRACT
}

\author{
Rajmohan, D $^{1 *}$,, D. Saranya ${ }^{1}$, K. Logankumar ${ }^{1}$, R. Ranjithkumar ${ }^{2}$ and B. Chandrashekar ${ }^{3}$ \\ ${ }^{1}$ Department of Zoology, Kongunadu Arts and Science College, Coimbatore. \\ ${ }^{2}$ Bionanotechnology research center, The Nilgiris Education and Research Foundation, The Nilgiris. \\ ${ }^{3}$ Department of Physics, Nanotechnology Research Lab, Kongunadu Arts and Science College, Coimbatore. \\ *E.mail: rajmohandevadass@gmail.com
}

\begin{abstract}
Biomimetic route for the synthesis of silver nanopartilces with using biological source play a very important role in nanotechnology without any harmful chemical. The present study deals with the synthesis of silver nanopartilces by treating silver nitrate with aqueous extract Vinca rosea at room temperature. The effect of the Vinica rosea aqueous extract on the formation of silver nanopartilces was characterized by UVvisible spectroscopy (UV-Vis), X-ray Diffraction Spectrum (XRD), Scanning Electron Microscopy (SEM) and Energy Dispersive X-ray Analysis (EDX). The UV spectra results show a strong resonance centre and surface of silver nanopartilces (AgNPs) at $461 \mathrm{~nm}$. XRD and SEM studies revealed that the synthesized AgNPs shows spherical in shape with average particles size around $30-70 \mathrm{~nm}$.
\end{abstract}

Keywords: Vinica rosea, AgNPs, SEM, XRD, UV-Vis, EDX.

\section{INTRODUCTION}

In the modern material science, nanotechnology plays a remarkable role with its eminent salient features such as manipulating nanoscale structures, engineering of atoms and designing of materials with improved properties (Jain et al., 2009). Nano-scale particles with size range of 1-100 $\mathrm{nm}$ and different shapes were commonly synthesized either by top-down or bottom-up strategies. At present development of reliable green chemistry route to synthesis nanoparticles is essential for their potential applications in diverse fields, specifically in biology and medicine (Narayanan and Sakthivel, 2011). The nanoparticles are synthesized through physical, chemical and biological methods (Chen et al., 2008). The physical and chemical methods are extremely pricey (Li et al., 1999). The biological methods of nanoparticles synthesis would assist to remove ruthless processing conditions, by allowing the synthesis at physiological $\mathrm{pH}$, temperature, pressure, and at the same time, at negligible cost. Huge number of micro organisms have been found competent of synthesizing inorganic nanoparticles composite, either intra or extracellularly. Ranjithkumar et al. (2013) reported plant extract based silver nanoparticles and achieved good antibacterial activity against human pathogens. Due to implausible properties, nanoparticles have turned into noteworthy in many fields in the recent years, such as energy, health care, environment, agriculture, etc. (Raveendran et al., 2003).
On other hand, nanotechnology is now creating a growing sense of excitement in the life sciences especially biomedical devices and Biotechnological applications (Prabhu et al., 2010). It has been reported that silver nanoparticles (SNPs) are non-toxic to humans and most effective against bacteria, virus and other eukaryotic micro-organism at low concentrations and without any side effects (Jeong et al., 2005). Moreover, several salts of silver and their derivatives are commercially manufactured as antimicrobial agents (Krutyakov et al., 2008). Sharma et al. (2009) report suggested in small concentrations, silver is safe for human cells, but lethal for microorganisms. Antimicrobial capability of silver nanoparticles allows them to be suitably employed in numerous household products such as textiles, food storage containers, home appliances and in medical devices (Marambio-Jones et al., 2010). The objectives of this study were to synthesize silver nanoparticles using Vinca rosea aqueous extract.

\section{MATERIALS AND METHODS}

\subsection{Materials}

The chemical silver nitrate $\left(\mathrm{AgNO}_{3}\right)$ was purchased from SD Fine Chemical Pvt. Ltd., Mumbai.

\subsection{Plant material}

The leaf of Vinca rosea was collected from Coimbatore district, Tamilnadu, India. The plant was identified Vinca rosea at the Department of Botany, 
Kongunadu Arts and Science College, Coimbatore, Tamilnadu, India.

\subsection{Preparations of the extract}

The leaf of Vinca rosea (V. rosea) was collected from Coimbatore district, Tamilnadu, India. The $V$. rosea plant leaf was washed with tap water followed by rinsed with distilled water thoroughly to remove dust and other any attached particles. The $V$. rosea aqueous leaf extract was prepared by taking $15 \mathrm{~g}$ of thoroughly washed and finely cut $V$. rosea leaves in a $250 \mathrm{ml}$ Erlenmeyer flask with $100 \mathrm{ml}$ of sterile distilled water and then boiled the mixture for 30 min at $60^{\circ} \mathrm{C}$ to obtain bioorganic compounds from $V$. rosea leaves. Followed by this step, after heating treatment, the solution was then removed from the heat source and left at room temperature and obtained aqueous extract was then filtered through a normal filter paper followed by Whatman filter paper No. 1. The final filtrate of the $V$. rosea leaf extract was used as a reducing agent to synthesis biomimetic silver nanoparticles.

\subsection{Synthesis of silver nanoparticles}

The aqueous solution of silver nitrate $\left(\mathrm{AgNO}_{3}\right)$ at concentration of $1 \mathrm{mM}$ was prepared to synthesize biomimetic silver nanoparticles from aqueous extract of $V$. rosea leaf. In details, $500 \mathrm{ml}$ of aqueous solution of at the concentration of $1 \mathrm{mM}$ silver nitrate solution was added to $25 \mathrm{ml}$ of $V$. rosea aqueous leaf extract while stirring for reduction into silver ions and the reaction mixture was kept at room temperature for $24-48 \mathrm{~h}$. The formation of dark brown colour was observed after suitable incubation time at room temperature and lambda max was taken using UV-Visible spectroscopy (JASCO V-670). Then the biomimetic silver nanoparticles solution was purified by repeated centrifugation at $6,000 \mathrm{rpm}$ for $20 \mathrm{~min}$ to isolate pure biomimetic silver nanoparticles free from other bioorganic compounds that present in the reaction mixture. After centrifugation the obtained particles were several time washed with distilled water for 10 to $20 \mathrm{~min}$ and kept it in Hot air oven for drying at $50^{\circ} \mathrm{C}$ for $3 \mathrm{~h}$ to obtain powder form of silver nanoparticles.

\subsection{Characterization Techniques}

\subsubsection{UV-Visible spectroscopy}

Formation of maximum production of biomimetic silver particles after $48 \mathrm{~h}$ incubation at room temperature the reaction mixture was confirmed by the colour change of the solution and the surface plasmon resonance band was obtained by UV-Visible spectral analysis which was done by using UV-Visible spectrophotometer (JASCO, V-670) from $300-800 \mathrm{~nm}$ at a resolution of $1 \mathrm{~nm}$.

\subsubsection{X-ray Diffraction spectrum}

X-ray diffraction (XRD) measurement of the biomimetic synthesized silver nanoparticles which were carried out using X'Pert Pro X-ray diffractometer (PAN analytical BV, The Netherlands) equipped with $\mathrm{Cu} / \mathrm{K \alpha}$ radiation source using $\mathrm{Ni}$ as filter at a setting of $30 \mathrm{kV} / 30 \mathrm{~mA}$. All X-ray diffraction data were collected under the standard experimental conditions in the regular angular range. The crystalline silver nanoparticles was calculated from the width of the XRD peaks, using a Debye-Scherer formula,

$$
\mathrm{D}=\frac{0.94 \lambda}{\beta \cos \theta}
$$

Where D is the average crystallite domain size perpendicular to the reflecting planes, $\lambda$ is the $\mathrm{X}$ ray wave length, $\beta$ is the full width at half maximum and $\theta$ is the diffraction angle.

\subsubsection{Scanning Electron Microscopy}

The reaction solution containing silver nanoparticles synthesis using $V$. rosea aqueous leaf extract was centrifuged at $6,000 \mathrm{rpm}$ for $20 \mathrm{~min}$. The supernatants were discarded and the final pellets were dissolved in $1 \mathrm{ml}$ of deionized water. The pellet was mixed properly and carefully placed on a glass cover slip followed by air-drying. The cover slip itself was used during scanning electron microscopy (SEM) analysis. The images of biomimetic silver nanoparticles were obtained in a SEM (Fb-Quanta 200 SEM machine) at different magnification level.

\subsubsection{Energy-dispersive X-ray (EDX) analysis}

The synthesized silver nanoparticles using $V$. rosea aqueous leaf extract subject to the Energy dispersive spectrum using SEM attached Fb-Quanta200 resolution to confirm the presence of silver in the particles as well as to detect other elementary compositions of the particle.

\section{RESULTS AND DISCUSSION}

A wide range of metabolites are presented in the plant extracts, nanoparticles produced by plants are more stable and the rate of synthesis is faster in comparison to other biological sources such as microorganisms. Thus, the advantages of using plant and plant derived materials for biosynthesis of metal nanoparticles have instigated researchers to investigate mechanisms of metal ions uptake and bioreduction by plants, and to understand the possible mechanism of metal nanoparticles 
formation in and by the plants (Naheed and Seema, 2012). In the present study the aqueous silver nitrate solution was reducing during exposure to the $V$. rosea (Fig. 1) plant leaf aqueous extract at $24-48 \mathrm{~h}$ incubation at normal room temperature.

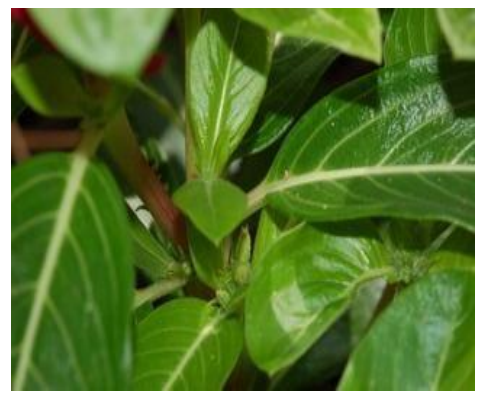

Fig. 1. The leaf of Vinca rosea

The colour of the reaction mixture changed from glow yellow to dark reddish brown after $48 \mathrm{~h}$ incubation at room temperature which indicates the formation of silver nanoparticles using $V$. rosea leaf aqueous extract. It is well known that silver nanoparticles exhibit dark brown colour in water due to excitation of surface Plasmon vibration in metal nanoparticles (Abhishek et al., 2014). Control (without silver nitrate) shows no colour change, when the $V$. rosea leaf aqueous extract with aqueous silver nitrate solution when incubated at 24-48h showed reddish brown colour this indicated the synthesis of silver nanoparticles (Fig. 2) due to present of bioactive compounds in aqueous extract of $V$. rosea leaf responsible for the reduction of silver nitrate to silver nanoparticles (Ranjithkumar et al., 2013). The different type of antioxidant and phytochemical are present in V. rosea plant extract, these phytochemical are responsible for the reduction of silver ions (Jayakumar et al., 2010).

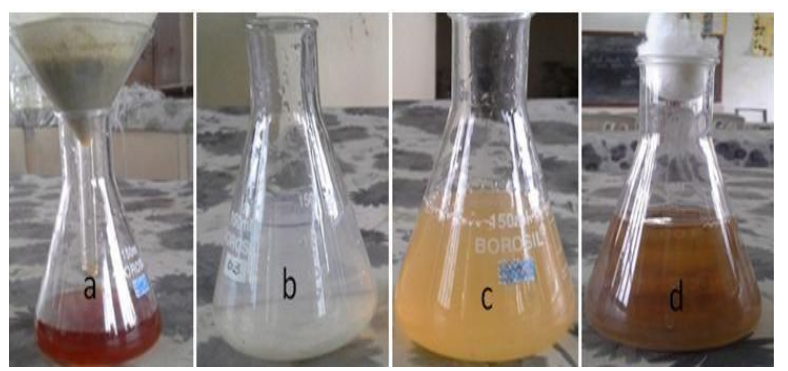

Fig. 2. Visual appearances of flask containing the aqueous extract of $\mathrm{V}$. rosea leaf and $\mathrm{AgNO}_{3}$ solution after $48 \mathrm{~h}$ reaction time

a) aqueous extract of $V$. rosea leaves b) $1 \mathrm{mM} \mathrm{AgNO}_{3} \mathrm{c}$ ) reaction mixture $0^{\text {th }} \mathrm{min}$ incubation d) reaction mixture after $48 \mathrm{~h}$ incubation at room temperature. The change of colour is an indication of the production of biomimetric silver nanoparticles.

\subsection{UV-visible spectroscopy analysis}

Formation of silver nanoparticles (AgNPs) by reduction with silver nitrate $\left(\mathrm{AgNO}_{3}\right)$ by aqueous extract of $V$. rosea leaf after $48 \mathrm{~h}$ incubation samples were characterized by using UV-Visible spectroscopy (JASCO-V/670) and this technique has proved to be very useful for the analysis of biomimetric silver nanoparticles formation in the reaction mixture. In the UV-Vis absorption spectrum, a strong, broad peak located between 420 to $471 \mathrm{~nm}$ was observed (Fig. 3). UV-Visible spectra also revealed that formation of AgNPs occurred rapidly within the 48 hours only and the AgNPs in solution remained stable even after 48 hour of completion of reaction. The similar type of the silver nanoparticles peaks were reported in Geranium leaf extract (Shankar et al., 2003), aqueous extract of areca nut (Ranjithkumar et al., 2013) and pomegranate peel extract (Shanmugavadivu et al., 2014). In this present study, the synthesized silver nanoparticles (AgNPs) were shown characteristic peak at 461 in visible light regions.

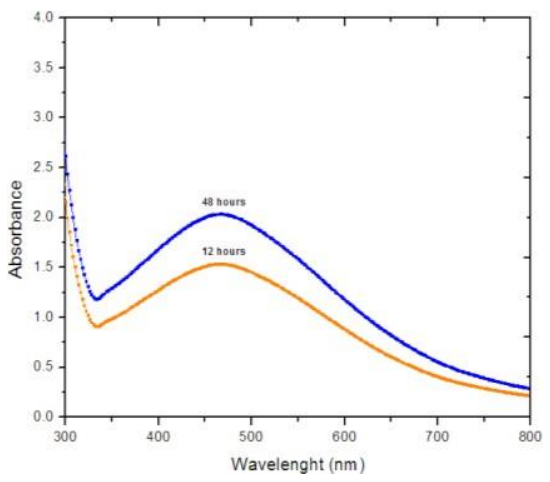

Fig. 3. Absorption spectrum of AgNPs synthesized by aqueous extract of Vinca rosea.

\subsection{XRD analysis}

Fig. 4 shows the X-ray diffraction spectrum of biomimetric synthesized AgNPs. The Braggs reflections were observed in the XRD pattern at $2 \theta$ $=32.27,46.25,59.60$ and 78.69 These Braggs reflections clearly indicated the presence of (200), (202), (311) and (402) sets of lattice planes and further on the basis that they can be indexed as facecentered-cubic (FCC) structure of silver. (Debabrat et al., 2012) reported that the XRD pattern green synthesized silver nanoparticles showed number of Braggs reflections that may be indexed on the basis of the face centered cubic structure of silver. Since, the present study clearly indicated the $x$-ray diffraction pattern of biomimetic synthesized silver nanoparticles formed crystalline in nature. Additional as yet unassigned peaks were also observed and suggesting that the crystallization of 
bioorganic phase occurred on the surface of the nanoparticles (Zuzer and Hemlatta, 2012).

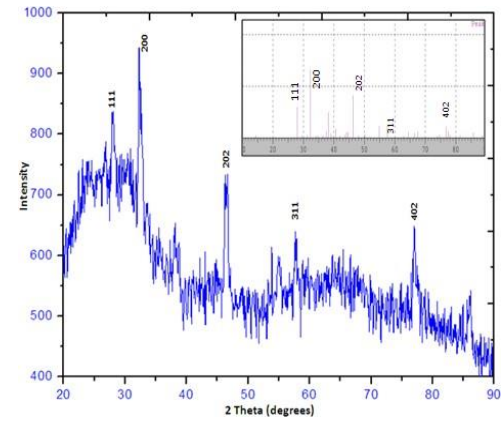

Fig. 4. X-ray diffraction spectrum of silver nanoparticles synthesized by aqueous extract of $V$. rosea leaf.

\subsection{SEM image}

The morphology of nanoparticles was determined by scanning electron microscope. The image of scanning electron microscopic of aqueous extract $V$. rosea plant leaf medicated biomimetric synthesized AgNPs shows high aggregation of silver particles on the surface of the cell. The XRD and SEM analysis revealed that the green synthesized silver nanoparticles were shown spherical in shape with particles size below $40-80 \mathrm{~nm}$ in diameter. The larger silver nanoparticles may be due to the high aggregation of the smaller ones. This may be due to availability of different quantity and nature of bioorganic compounds present in the aqueous extract of $V$. rosea leaf. Fig. 5 shown different magnification scanning electron microscopic images of biomimetric synthesized silver nanoparticles using V. rosea leaf extract. Previous observations indicated that the plant phytochemicals may be responsible for bioreduction of $\mathrm{Ag}^{+}$to $\mathrm{Ag}^{0}$ and subsequent formation of silver nanoparticles, the obtained AgNPs shown spherical in shape with high aggregation (Ranjithkumar et al., 2013; Shanmugavedivu et al., 2014).

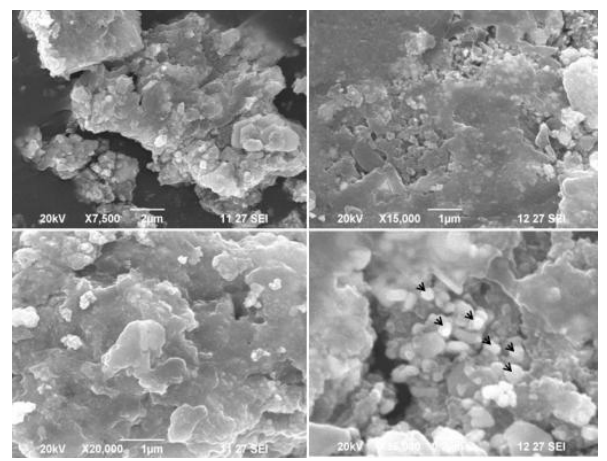

Fig. 5. SEM image of biomimetic synthesized AgNPs

\subsection{EDX analysis}

Energy-dispersive X-ray spectroscopy (EDX) is an analytical technique used for the elemental analysis or chemical characterization of a sample. In this present study, the element analysis of the biomimetic synthesized AgNPs was performed using EDX spectrum (Fig. 6). The EDX spectrum of spherical in shape with high aggregation of silver nanoparticles on the surface of the cell prepared with this bioreduction method using $V$. rosea shown maximum peaks around $3.28 \mathrm{keV}$ correspond to the binding energies of silver ions. Throughout the scanning range of binding energies, some addition peaks belonging to bioorganic compound present in the reaction mixture. The EDX analysis revealed strong signals in the silver region and confirms the formation of silver nanoparticles by using biological source. There were other EDX spectrum peaks for $\mathrm{Cl}$, $\mathrm{Si}, \quad \mathrm{O}$ and $\mathrm{Ca}$ suggesting that they are mixed precipitates present in the plant extract (Usha and Gladys, 2014).

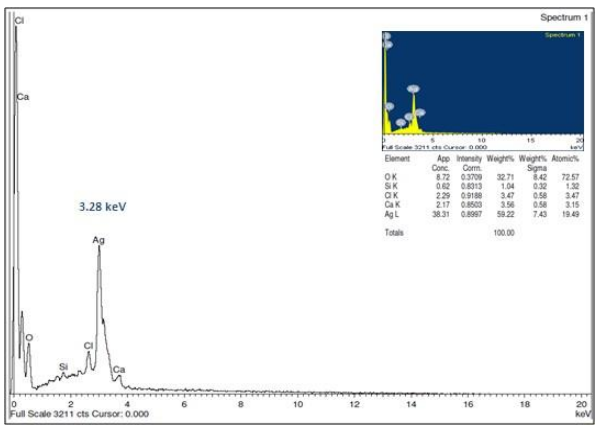

Fig. 6. EDX spectrum of prepared AgNPs using $V$. rosea at room temperature.

\section{CONCLUSION}

A simple and environmental free green route was used to synthesis AgNPs from silver nitrate using aqueous extract of Vinca rosea leaf. The effect of the Vinica rosea aqueous extract on the formation of silver nanopartilces after 48 incubation showed reddish brown colour. From UV-Vis spectrum indicated the synthesized AgNPs were shown characteristic peak at 461 in visible light regions. From XRD and SEM studies revealed that the synthesized AgNPs shows spherical in shape with average particles size round around $30-70 \mathrm{~nm}$. From the present work the aqueous extract of Vinca rosea leaf was appropriate for synthesis silver nanopartilces at room temperature.

\section{REFERENCES}

Abhishek, M., Akhilesh Kushwaha, Vandana Dalakoti, Garima Dalakoti and Deep Shikha Singh, (2014). Green synthesis of silver nanoparticles using 
medicinal plant and its characterization. Der.

Pharmacia. Sinica. 5(5): 118-122,.

Chen, H., M.C. Roco, X. Li and Y. Lin, (2008). Trends in nanotechnology patents. Nat. Nanotechnol. 3: 123-125.

Debabrat, B., Nakul Sharma and Rituparna Bora, (2012). Green Synthesis of Silver Nanoparticle using Bryophyllum pinnatum (Lam.) and monitoring their antibacterial activities, Arch. Appl. Sci. Res. 4(5): 2098-2104,.

Jain, D., H.K. Daima, S. Kachhwaha, S.L. Kothari and J. Digest, (2009). Nanomater. Biostruct. 4: 723727.

Jayakumar, D., S. Jhancy Mary and R. Jaya Santhi, (2010). Evaluation of antioxidant potential and antibacterial activity of Calotropis gigantea and Vinca rosea using in-vitro model, Ind. J. Sci. Technol. 3(7): 720-723,.

Jeong, S.H., S.Y. Yeo and S.C. Yi, (2005). The effect of filler particle size on the antibacterial properties of compounded polymer/ silver fibers. J. Mat. Sci. 40: 5407-5411.

Krutyakov, Y.A., A. Kudrynskiy, A.Y. Olenin and G.V. Lisichkin, (2008). Extracellur biosynthesis and antimicrobial activity of silver nanoparticles. Russ. Chem. Rev. 77: 233.

Li, Y., X. Duan, Y. Qian, L. Yang and H. Liao, (1999). Nanocrystalline silver particles: synthesis, agglomeration, and sputtering induced by electron beam. J. Colloid Interface Sci. 209: 347349.

Marambio-Jones, C. and E.M.V. Hoek, (2010). A review of the antibacterial effects of silver nanomaterials and potential implications for human health and the environment. $J$. Nanopart. Res. 12: 1531-1551.

Naheed Ahmad and Seema Sharma, (2012). Green Synthesis of Silver Nanoparticles Using Extracts of Ananas comosus. Green Sus. Chem. 2: 141-147.
Narayanan, K.B. and N. Sakthivel, (2011). Adv. Colloid Interface Sci. 169: 59-79.

Prabhu, N., T.R. Divya and G. Yamuna, (2010). Synthesis of silver phyto nanoparticles and their antibacterial efficacy. Digest. J. Nanomater. Biostruct. 5: 185-189.

Ranjithkumar, R., Selvam Kuppusamy and M. Shanmugavadivu, (2013). Green synthesis of silver nanoparticles using areca nut extract for enhanced antibacterial activity, J. Green Sci. Technol. 1: 102-106.

Raveendran, P., J. Fu and S.L.Wallen, (2003). Completely "green" synthesis and stabilization of metal nanoparticles. J. Am. Chem. Soc. 125: 13940-13941.

Shankar, S.S., A. Ahmad and M. Sastry, (2003). Geranium leaf assisted biosynthesis of silver nanoparticles, Biotechnol. Prog. 19(6): 16271631.

Shanmugavadivu, M. Selvam Kuppusamy and R. Ranjithkumar, (2014). Synthesis of pomegranate peel extract mediated silver nanoparticles and its antibacterial activity. $\mathrm{Am}$. J. Adv. Drug Del. 2(2): 174-182.

Sharma, V.K., R.A. Yngard, Y. Lin, (2009). Silver nanoparticles: Green synthesis and antimicrobial activities. Adv. Coll. Int. Sci. 145: 83-96.

Usha, C. and D. Gladys Angelin Rachel, (2014). Biogenic synthesis of silver nanoparticles by Acacia nilotica and their antibacterial activity. Int. J. Scientific Res., 3(6): 27-29.

Zuzer, H.D. and Hemlatta Chakraborty, (2012). Lactobacillus mediated synthesis of silver oxide nanoparticles, Nanomater. Nanotechnol. 2(15): 1-7. 